\title{
Drug resistance in lung cancer
}

\author{
This article was published in the following Dove Press journal: \\ Lung Cancer:Targets and Therapy \\ 8 May 2010 \\ Number of times this article has been viewed
}

\author{
Manish Shanker \\ David Willcutts \\ Jack A Roth \\ Rajagopal Ramesh \\ Department of Thoracic and \\ Cardiovascular Surgery, The \\ University of Texas M D Anderson \\ Cancer Center, Houston, Texas, USA
}

Correspondence: Rajagopal Ramesh

Department of Thoracic and

Cardiovascular Surgery, Unit 445,

The University of Texas M D Anderson

Cancer Center, 1515 Holcombe

Boulevard, Houston, TX 77030, USA

$\mathrm{Tel}+$ I 7135639144

Fax + I 713794 490।

Email rramesh@mdanderson.org
Abstract: Resistance to chemotherapy drugs is a major problem in cancer treatment. Scientific advances made in the last two decades have resulted in the identification of genes and molecular signaling mechanisms that contribute to drug resistance. This has resulted in a better understanding of the biology of cancer cells and the way these cells adapt or undergo subtle molecular changes thereby protecting themselves from the cytotoxic effects of the anticancer drugs. Based on the knowledge gained to-date new molecularly targeted drugs are being developed and tested in clinical studies, in an attempt to overcome drug resistance and improve drug efficacy. Despite these attempts the overall 5-year survival of patients diagnosed with cancer, such as lung cancer, remains dismal and is less than $15 \%$. It is evident that additional mechanisms contributing to drug resistance exist which are yet to be discovered. It is hoped that identification of new targets and understanding their contribution to drug resistance will provide opportunities for innovative therapies in overcoming drug resistance. In an attempt to broaden our knowledge and understanding on drug resistance we have, in this review article, summarized the most common mechanisms associated with drug resistance in lung cancer.

Keywords: lung cancer, chemotherapy, resistance, apoptosis, proteasome, DNA damage

\section{Introduction}

Chemotherapy is a major treatment modality in both primary and palliative care of patients with lung cancer. Some patients' disease does not respond to such therapy, or it responds well initially and then gradually relapses. This may lead to an increase in the drug dosage, which generally increases the adverse affects, yet fails to improve the clinical prognosis or outcome.

Drug resistance is associated with a wide variety of solid tumors. In this review, however, we focus on the role of resistance in lung cancer. Non-small cell lung cancer (NSCLC) cells are often intrinsically resistant to certain anticancer drugs, whereas small-cell lung cancer (SCLC) cells can acquire resistance with continued administration of the drug. Moreover, at the time of diagnosis, the majority of patients with lung cancer most often already have metastatic disease, making it difficult to use other therapeutic options, such as surgery and radiation. Thus, a better understanding of the different mechanisms underlying drug resistance is of utmost importance if we are to develop strategies to overcome it.

Although numerous mechanisms are associated with drug resistance in lung cancer, we are a long way from fully understanding how to overcome drug resistance. Mechanisms identified so far include increased drug efflux, drug inactivation and/or sequestration

submit your manuscript $\mid$ www.dovepress.com 
by enzymes, DNA repair, target modifications, and apoptosis defects. ${ }^{1-5}$ Additional contributing factors include ineffective drug delivery to the tumor, increased metabolism and therefore a shortened half-life of the drug, lack of drug specificity to the tumor, and tumor vasculature. 6,7 These factors make it even harder to pinpoint the exact mechanism underlying resistance to a particular drug.

To further complicate our understanding of drug resistance, patients given chemotherapy gradually develop genetic mutations with each clonal expansion of the tumor cell. These mutations may result from either activation of proto-oncogenes or inactivation of tumor-suppressor genes. This continuous genomic instability eventually leads to tumor progression and metastatic changes, making treatment difficult in such patients; coexisting drug resistance of the tumors makes it even more difficult to treat the primary and metastatic lesions. Moreover, tumors that are resistant to one particular drug are either already cross-resistant or develop resistance to other chemotherapy drugs fairly quickly. Indeed, SCLC, for example, initially responds to chemotherapy, but the patients invariably experience a relapse, and the tumor becomes resistant to chemotherapeutic treatment. Therefore, overcoming drug resistance in lung cancer has remained a challenge resulting in a poor 5-year survival rate that remains less than $15 \%$ for NSCLC and 5\% for SCLC.

In this review, we focus on the major pathways and mechanisms that have been shown to contribute to drug resistance in lung cancer.

\section{Drug transporters involved in the efflux of chemotherapeutic drugs}

Drug transporter proteins can be classified according to their mechanism of action, ATP dependency, and pharmacodynamics and/or pharmacokinetics. One class of transporters, known as the ATP binding cassette (ABC) superfamily, plays a major role in drug resistance. The $\mathrm{ABC}$ proteins consist of seven subclasses, namely $\mathrm{ABCA}$ to $\mathrm{ABCG}$. Overexpression of $\mathrm{ABC}$ transporters can lead to drug efflux and decreased intracellular accumulation, thereby conferring resistance to drugs such as cisplatin, methotrexate, taxanes, anthracyclines, and vinca alkaloids.

The ABC family proteins, such as P-glycoprotein (P-gp), multidrug resistance-associated proteins (MRPs) and breast cancer-resistance protein which belong to the $\mathrm{ABCB}, \mathrm{ABCC}$ and $\mathrm{ABCG}$ family of $\mathrm{ABC}$ transporters respectively, have been shown to play a major role in drug resistance. Additionally, transporter proteins such as major vault protein (MVP), also known as the lung resistance-related protein (LRP) have been implicated in drug resistance in lung cancer.
P-gp, the product of the $m d r l$ gene, is a $170-\mathrm{kDa}$ membrane protein that acts as a drug efflux pump and is up-regulated in numerous drug-resistant cancer cell lines. Studies have shown a high expression of P-gp in lung tumors, notably higher in NSCLCs than in SCLCs. Other studies have shown that lung tumor cell lines that were resistant to vincristine demonstrated cross-resistance to doxorubicin. ${ }^{8}$ Molecular analysis revealed that these drug-resistant cell lines had a higher expression of both P-gp and the $m d r 1$ gene than did the parental cell lines. In addition, in vitro studies demonstrated a higher expression of P-gp in NSCLC cell lines from smokers. ${ }^{9}$

A further role of $m d r l$ in drug resistance was demonstrated by gene array studies where the expression of various genes were analyzed in paclitaxel sensitive (NCI-H460) and resistant (NCI-H40/PTX250) human large-cell lung carcinoma cell lines. The results indicated increased expression of 332 genes and decreased expression of 342 genes in the NCI-H460/PTX250 cell line as compared to the NCI-H460 sensitive cell line, with a 1,092 fold increase in the expression of MDR1 observed in the paclitaxel resistant cell line. ${ }^{10}$

On the basis of these and other study results, drugs targeting the $m d r l$ gene or P-gp have been developed to overcome P-gp-associated drug resistance. ${ }^{11}$ In a recent study, P-gp inhibition in a mouse model of paclitaxel-resistant tumor yielded greater inhibition of tumor growth when the mice were treated with biotin-functionalized nanoparticles encapsulating both paclitaxel and P-gp-targeted small interfering RNA than when they were treated with paclitaxel alone. Thus, silencing the $P-g p$ gene may be one way to overcome drug resistance in cancers over expressing the $\mathrm{P}$-gp protein. ${ }^{12} \mathrm{How}-$ ever, Helfrich and colleagues have shown that both NSCLC and SCLC cell lines established from patients with lung cancer who had previously been treated with chemotherapy expressed low levels of MDR-1 or showed no significant increase in MDR-1 expression. ${ }^{13}$ These results thus question the contributing role of $m d r 1$ and P-gp in drug resistance in lung cancer and warrant further investigation.

Although P-gp has been shown to play a major role in drug resistance, other non-P-gp mediated mechanisms attributed to proteins such as MRP1 have also been implicated in NSCLC drug resistance. ${ }^{14}$ To date, seven isoforms of MRP have been identified (MRP1-MRP7). The major isoforms that have been shown to play a role in drug resistance are MRP1, MRP2, MRP3 and MRP5. ${ }^{15}$ Comparative studies performed with a panel of 30 lung cancer cell lines showed that NSCLC cell lines expressed higher levels of MRP3, MRP1, and MRP2 protein than SCLC cell lines did. Furthermore, it was noted 
that expression of both MRP3 and MRP1, but not MRP2, correlated with decreased sensitivity to doxorubicin, VCR, VP-16, and cisplatin in those cell lines, suggesting that MRP has a role in producing drug resistance in lung cancer. ${ }^{16}$

Similarly, a study conducted to analyze the expression of $\mathrm{ABC}$ transporter protein in relation to vinolrelbine in NSCLC demonstrated higher expression of ABCC10/MRP7 in 12 out of 17 lung cancer cell lines. Furthermore, this increase in expression was correlated with resistance to vinolrelbine, thereby demonstrating involvement of other ABC transporter proteins other than MDR-1 in attributing to drug resistance. ${ }^{17}$ Another study demonstrated the involvement of a non-ABC transporter protein, RLIP 76 in contributing to vinolrelbine resistance. RLIP76 is a transporter involved in catalyzing energy-dependent efflux of various chemotherapeutic drugs, such as doxorubicin. ${ }^{18,19}$ Thus resistance to a chemotherapeutic drug may not be due to one particular $\mathrm{ABC}$ transporter protein but may be due to a defect in multiple transporter proteins. ${ }^{20}$

MVP was first discovered in an NSCLC cell line that was selected for doxorubicin resistance. ${ }^{21}$ Vaults are huge intracellular ribonucleoprotein particles that are involved in shuttling proteins and in redistributing drugs such as doxorubicin from the nucleus to the cytoplasm, thereby leading to resistance. ${ }^{22,23}$ LRP expression correlates with chemoresistance in a broad range of tumor cell lines. ${ }^{22}$ A study involving NSCLC and SCLC cell lines demonstrated that the expression of LRP was higher in NSCLC than in SCLC samples, with the highest expression in squamous cell and adenocarcinomatous histology type of NSCLC. ${ }^{19}$ However, in the same study the authors demonstrated LRP expression did not predict chemotherapeutic response in lung cancer patients. ${ }^{24}$ Thus, additional studies are needed to further evaluate the role of LRP in lung cancer drug resistance.

\section{Drug inactivation by sulfur- containing macromolecules and role of antioxidants as a cause of drug resistance}

Although drug efflux is a major mechanism associated with drug resistance, another mechanism by which cancer cells avoid the drug is by conjugation of the drug with sulfur-containing macromolecules such as metallothioneins (MTs) and glutathione (GSH). Furthermore, antioxidants such as, superoxide dismutase (SOD) have been shown to be implicated in drug resistance.
The MTs are low molecular-weight intracellular proteins with a high cysteine content that can bind to several heavy metal ions such as zinc, copper, selenium, cadmium, platinum, and mercury. MTs can also bind to drugs such as cisplatin, thereby leading to decreased sensitivity to cisplatin in SCLC. ${ }^{25,26}$ It has also been suggested that MTs play a role in protecting cells from oxidative stress and metal toxicity. ${ }^{27,28}$ Since numerous drugs lead to cancer cell death by inducing oxidative stress and metal toxicity; overexpression of MTs in such cells may also contribute to drug resistance. To date, four different isoforms of methallothionein (MT-1 through MT-4) have been identified. MT-1 and MT-2 are the most widely expressed, with the MT isoform 2a (MT-2a) being the most widely distributed MT in humans. MT-3 localizes mostly in the brain but has also reportedly been found in other tissues, such as the stomach, heart, and kidneys. ${ }^{29,30}$ MT-4 has been detected in certain stratified squamous epithelia. ${ }^{31}$ The expression of MTs can be induced by several metals, cytokines, stress hormones, and chemicals. ${ }^{32}$ Furthermore, it is overexpressed in various tumors such as colorectal, breast, testicular, and ovarian. ${ }^{33-35}$ MT expression is also higher in tumors with high metastatic and proliferative potential. ${ }^{36}$ Although some investigators have found relatively low levels of MTs in some cancers, such as gastrointestinal cancers, they also found that higher expression of MT correlates with a greater metastatic potential. ${ }^{37}$

In lung cancer, the high expression of MT was observed in NSCLC with histologic features of squamous cell lung carcinoma and adenocarcinoma but was not observed in SCLC. ${ }^{38}$ Contradictory to this report Kasahara and colleagues reported a correlation between MT expression and cisplatin resistance in SCLC cell lines in vitro. In this study the SCLC cell line, H69 was made resistant to cisplatin treatment and then analyzed for the expression of both intracellular MT protein and mRNA in resistant and parental cell lines. Increased MT expression at both mRNA and protein level were observed in cisplatin resistant $\mathrm{H} 69$ cells compared to the parental cell line. ${ }^{25}$ The conflicting reports suggest additional studies, analyzing MT expression and drug resistance in SCLC, which are more detailed are warranted in SCLC. Correlation between high MT expression and cisplatin resistance have also been reported for other tumor types. ${ }^{39}$ Apart from cisplatin resistance, a strong correlation between MT expression and doxorubicin resistance has been reported in SCLC cell lines. ${ }^{40,41}$ Thus, although there seems to be a significant correlation between MT expression and drug resistance in lung tumors, further studies are warranted to completely explore the mechanisms underlying MT overexpression in lung cancer. 
Other inactivating enzymes that are often up-regulated in drug-resistant cell lines include the glutathioneS-transferases (GSTs), a family of phase II detoxification enzymes. ${ }^{42}$ GSTs protect the cancer cells from reactive endogenous and exogenous electrophiles, such as prostaglandins, aromatic hydrocarbons, and chemotherapy agents, by conjugating those compounds to the sulfur containing macromolecule glutathione-S-hydroxylase (GSH), thus detoxifying them. The GSTs fall into either the membranebound microsomal family or the cytosolic family. In turn, the cytosolic GSTs have six isoforms that are named with the Greek letters $\alpha, \pi, \mu, \omega, \theta$, and $\zeta$. The $\pi$ and $\mu$ isoforms also play a role in regulating the mitogen-activated protein (MAP) kinase (MAPK) pathway.

Therefore, the distinct role of GSTs in drug resistance involves two different mechanisms, drug detoxification and MAPK pathway inhibition. Many chemotherapeutic drugs that form electrophilic moieties are detoxified through the GSH mechanism, thereby leading to resistance. This may also explain the increase in GST levels observed over time with continued chemotherapy treatment - it is an attempt to detoxify the drug. However, drugs that are not conjugated to GSH can still inhibit the MAPK kinase pathway, thereby protecting cells from chemotherapy drugs that specifically target this pathway. Numerous drugs used in lung cancer treatment, such as cisplatin, kill tumor cells by inducing apoptosis by activating the JNK/MAPK pathway. Inhibition of this pathway by GSTs leads to decreased cisplatin-induced apoptosis, thereby conferring resistance to the drug.

Although some investigators have reported finding expression of GST isoenzymes in the bronchioles and alveoli of normal lungs, ${ }^{43}$ studies have demonstrated greater expression of these isoenzymes in lung tumors. ${ }^{44}$ Furthermore, cell lines that are resistant to nitrogen mustard compounds, nitroureases, and doxorubicin exhibit a relatively high level of expression of GST- $\pi{ }^{45}$ Transfection of cells with GST complementary DNA showed that cell lines can become resistant to certain chemotherapy drugs, thereby demonstrating that GSTs themselves play an important role in contributing to drug resistance. ${ }^{46}$ In addition, other mechanisms, such as co-expression of P-gp or MRP1 with GST- $\pi$, may also contribute to drug resistance. ${ }^{9,47}$

Another mechanism by which cancer cells develop resistance is by overexpression of antioxidants that protect cells from chemotherapy-induced oxidative stress and cell death. Numerous chemotherapeutic drugs such as epirubicin cause oxidative damage by generation of superoxide and hydrogen peroxide moieties, thereby leading to cancer cell death. However, overexpression of antioxidants such as SOD and GSH can neutralize the drug-induced oxidative stress, thereby leading to drug resistance. Overexpression of SODs such as manganese SOD (MnSOD) can also protect lung epithelial cells against oxidant injury. ${ }^{48}$ Furthermore, cultured malignant mesothelioma cells reportedly have higher levels of both MnSOD messenger RNA (mRNA) levels and activities than nonmalignant mesothelial cells have. ${ }^{49,50}$ Additional study results have shown that, other antioxidant enzymes besides MnSOD, such as GSH and GST, can be involved in high hydrogen peroxide levels and thus resistance to epirubicin. ${ }^{50}$ Combined, these results suggest that antioxidant activities that protect tumor cells from cytotoxic drugs are attributable to overexpression of not just one but of multiple antioxidant enzymes in cells resistant to these drugs.

\section{DNA-repair pathways inducing resistance to chemotherapy}

The primary target for most lung cancer chemotherapeutic agents is cancer cell DNA. For instance, cisplatin induces apoptosis primarily by forming DNA-platinum adducts and by introducing oxidizing agents, thus causing oxidative damage to the DNA of cancer cells. ${ }^{51}$ With drugs such as cisplatin, the onset of tumor resistance to chemotherapy is caused partly by the inherent resistance to DNA damage that is common among tumors. The difficulty in dealing with resistance due to DNA-repair enzymes is that although there are many different drug-induced pathways of DNA degradation and damage, there are also many DNA-repair enzymes, each of which has different mechanisms of action. Furthermore, these sophisticated repair mechanisms are often up-regulated in resistant cancer cells, and each tumor has its own unique genetic profile of overexpressed repair enzymes.

The nucleotide excision-repair (NER) pathway is one DNA repair pathway suggested to be a common culprit in the emergence of platinum-based drug resistance. ${ }^{52}$ Excision-repair cross-complementation group 1 (ERCC1) protein, encoded by the ERCC1 gene is a key enzyme that is a molecular indicator of resistance to platinum-based chemotherapy; along with other proteins, ERCC1 forms the critical molecular complex of the NER pathway. ${ }^{53}$ This key molecular complex removes modified nucleotides, such as the DNA-platinum adducts, and adjacent nucleotides from the damaged DNA strand. ${ }^{54}$ This excision, for which the enzyme is named, essentially negates the primary method of cytotoxicity of platinum-based drugs. By removing the 
DNA adducts, DNA polymerase can repair and/or synthesize new DNA, and the tumor cell can divide normally again. In vitro studies have demonstrated an association between the expression of ERCC1 mRNA in NSCLC and resistance to the platinum drugs: low expression of ERCC1 correlated with prolonged survival of patients with NSCLC who were treated with cisplatin plus gemcitabine chemotherapy. ${ }^{55,56}$

A second DNA-repair pathway, the mismatch-repair (MMR) pathway, repairs base-base and insertion-deletion mismatches generated during DNA replication or recombination of DNA. ${ }^{57} \mathrm{MMR}$ is usually a cancer-preventing pathway that helps to maintain genomic stability, but it can also lead to resistance against certain chemotherapeutic agents. ${ }^{58}$ For example, the MMR pathway can repair DNA-platinum adducts, although the attempted repair can result in mitotic crisis, which leads to cell death. ${ }^{59,60}$ Moreover, it often plays a key part in the apoptotic pathway of methylating agents, so much so that defects and/or deficiencies in the MMR pathway are associated with resistance to methylating agents. Overall, however, this repair pathway does not appear to be an important source of chemotherapy resistance in lung cancer.

The base excision-repair (BER) pathway is a third DNA-repair pathway that has been linked with chemotherapy resistance. When N-methylpurine DNA Glycosylase (MPG) and Apurinic/Apyrimidinic Endonuclease (APE), two molecular indicators of the BER pathway, are inhibited or eliminated in alkylating agent-resistant cancer cells, then sensitivity to alkylating agents such as platinum-based drugs increases. ${ }^{61}$ The BER pathway is initiated by DNA glycosylases, which recognize and catalyze the removal of damaged DNA bases. The pathway is then completed when the gaps between the bases are filled, which prevents the apoptotic affects of chemotherapy-induced DNA damage. ${ }^{62}$

A fourth DNA-repair pathway, the non-homologous endjoining (NHEJ) pathway, is the major pathway used for repairing double-strand breaks (DSBs) in human DNA. As such, it leads to resistance of cancer cells to multiple chemotherapy drugs and ionizing radiation by repairing DNA damage and stopping apoptosis. The NHEJ pathway begins when Ku proteins, which is a heterodimer composed of two polypeptides, $\mathrm{Ku} 70$ (XRCC6) and Ku80 (XRCC5) bind with the broken ends of DNA DSBs. The Ku protein functions as a molecular scaffold which helps in binding of other proteins that are involved in DNA repair by NHEJ pathway. This attracts DNA-dependent protein kinase catalytic subunit (DNAPKcs) to the DNA bound $\mathrm{Ku}$, which then brings the two, ends of the broken DNA together. The pathway is completed with the recruitment of proteins such as artemis, XRCC4/DNA ligase
IV, polynucleotide kinase (PNK), and Cernunnos-XRCC4 like factor (XLF), which process the ends of the DNA and fill the gaps between the DSBs. ${ }^{63-65}$ Inhibition of the NHEJ pathway by the drug trifluoperazine significantly lessens the resistance to the chemotherapy drug bleomycin. ${ }^{66}$ The drug doxorubicin also induces more apoptosis in cancer cells when those cells are deficient in DNA ligase IV or DNAPKcs; two of the integral proteins in the NHEJ pathway. ${ }^{67}$

The last major DNA-repair pathway to be discussed here is the homologous-recombination (HR) pathway. This pathway begins with the recruitment of proteins to form the $\mathrm{M} / \mathrm{R} / \mathrm{N}$ complex (consisting of Mre11, Rad50, and NBS1) together with recruitment of replication protein A (RPA), which is a single-stranded DNA-binding factor. These bind the broken ends of the DNA strands together and begin the process of homologous DNA pairing for which the HR is named. ${ }^{68,69}$ Furthermore, it should be noted that the HR pathway can repair the same kind of DNA-platinum adducts that occur in the NER pathway. ${ }^{70}$ Although these adducts do not constitute DSBs, ERCC1 (in the NER pathway), in conjunction with other proteins they can convert these adducts into DSBs, which can then be repaired by the HR pathway. ${ }^{70}$ This enables cancer cells to have two separate pathways for resisting the damage caused by alkylating agents such as the platinum drugs.

As for chemotherapeutic agents that induce DSBs, such as etoposide, study results showed that SCLC cells over expressing RAD51, a key protein in the HR pathway, were resistant to etoposide, whereas cells with relatively lower levels of RAD51 were more sensitive to the drug. ${ }^{71,72}$

Apart from these pathways other enzymes involved in DNA synthesis and repair have also been implicated in attributing resistance to drugs such as pemetrexed and gemcitabine that have been used for the treatment of lung cancer.

Pemetrexed, a folate anti-metabolite, is frequently used in the treatment of pleural mesothelicma and NSCLC. Thymidylate synthase (TS) is an enzyme involved in DNA synthesis and repair and has been associated with resistance to pemetrexed. It has been shown that higher expression of TS can lead to resistance to pemetrexed in colon cancer. ${ }^{73}$ However, increased levels of TS leads to resistance not only to pemetrexed but other antifolate drugs such as, Thymitaq and Raltitrexed (RTX). This increased resistance to pemetrexed was attributable to an increased expression of TS alone. Another study had similar results both in vitro and in vivo, when cells treated with 5-fluorouracil (5FU) and RTX led to a 2 to 5 fold increase in the expression of TS, thereby leading 
to an acquired resistance to these and other drugs. ${ }^{74}$ In vitro, treatment with 5 FU initially leads to decreased TS and then a 2- to 3-times induction. Similar results were obtained in patients who were treated with 5FU and their tumors were collected to analyze the expression of TS. There was an initial decrease in TS following 5-FU therapy followed by an induction. This makes it important to screen patients for TS expression prior to resuming chemotherapy.

Ribonucleoside-diphosphate reductase subunit M1 (RRM1) is another enzyme, involved in DNA synthesis and repair encoded by the RRM1 gene. This gene encodes the regulatory subunit of ribonucleoside-diphosphate reductase, an enzyme needed for deoxyribonucleotide production from ribonucleotides prior to DNA synthesis in the $\mathrm{S}$ phase. It has been shown that resistance to gemcitabine is attributed to RRM1; tensin homolog deleted on chromosome ten (PTEN) and ERCC1 in lung cancer. ${ }^{75}$ The mRNA expression of 6 lung cancer cell lines including human lung adenocarcinoma, giant cell and squamous cell carcinoma was analyzed using real time polymerase chain reaction (PCR). The results demonstrated higher expression levels of RRM1; PTEN and ERCC1 in these cell lines; with the highest expression in lung squamous cell line, QG56.

\section{Loss of intracellular death mechanisms as a cause of drug resistance}

The goal of chemotherapy is to induce either growth arrest or the killing of cells that are malignant or undergoing malignant transformation, while sparing normal cells. Many chemotherapy drugs induce cell death via the activation of apoptosis and other intracellular death mechanisms. Drugs such as cisplatin and carboplatin induce tumor cell death by damaging DNA; however, the end result of that is still activation of the apoptotic pathways. Thus, the loss of apoptotic function or other intracellular death mechanisms will eventually lead to tumor cell survival and progression of the cancer by making the cells resistant to chemotherapy.

Because multiple tumor-specific pathways are involved in cell death, it is difficult to identify the exact mechanism that a particular drug uses to kill tumor cells and to determine the exact cause of the lost apoptotic function that leads to drug resistance. However, various apoptotic and antiapoptotic intracellular proteins, such as Bcl-2, Bax, and SAPK/JNK, contribute to drug resistance caused by failure of the intracellular death signaling pathways. Drugs such as paclitaxel, which induce cell death by regulating cellular microtubule dynamics, kill cells in a Fas/Fas ligand
(FasL)-dependent manner. ${ }^{76}$ Thus, inhibiting Fas/FasL with an antibody leads to the abrogation of paclitaxel-induced cell killing. Furthermore, overexpression of Bcl-2 leads to the decreased expression of FasL, reduced apoptosis of tumor cells and thus resistance to paclitaxel. ${ }^{68}$ Srivastava and colleagues showed that phosphorylation of Bcl-2 induced the expression of FasL by allowing nuclear translocation of NFAT (nuclear factor of activated T lymphocytes), which is a transcription factor activated by microtubule damage to the nucleus; and initiating transcription of FasL gene, thereby restoring paclitaxel sensitivity. ${ }^{77}$ In contrast Inoue and coworkers showed that increased expression of Bcl-2 led to increased sensitivity to docetaxel in NSCLC cells. ${ }^{78}$ The discrepancy in the two reports could be due to several reasons and needs further validation.

In addition, drugs such as cytarabine, doxorubicin, and methotrexate, which activate the apoptotic pathways, reportedly can confer resistance to cancer cells if their caspases are defective. ${ }^{79}$ The use of antisense approaches targeting caspases 1 and 3 revealed that such tumor cells are protected from drug-induced cell death, indicating that the integrity of the caspase pathway is an important contributing factor to drug resistance. ${ }^{79}$ Moreover, chemotherapy-resistant NSCLC cells have a decreased expression of caspases 3 and 9, which contributes to the drug resistance; the restoration of caspase activity sensitized the tumor cells to cisplatin chemotherapy. ${ }^{80}$

Some in vivo studies have demonstrated a correlation of caspase-3 expression and the survival in NSCLC. A study using 70 squamous cell lung carcinoma demonstrated an inverse relationship between the expression of caspase-3, vascular endothelial growth factor (VEGF), and microvessel density, showing the importance of pro-apoptotic proteins in regulation of tumor growth. ${ }^{81}$

Defects in not only pro-apoptotic but also anti-apoptotic proteins, such as IAP (inhibitor of apoptosis protein) and survivin have been implicated in resistance in human NSCLC. IAPs are characterized by a novel domain of approximately 70 amino acids and were first in identified in baculoviruses. IAP's, as the name implies, inhibit apoptosis either by inhibition of the caspases or by ubiquitin-tagging and proteasomal degradation of certain proteins involved in apoptosis. To date eight different types of IAPs have been identified, namely: apollon/BRUCE, cIAP-1 (cellular inhibitor of apoptosis protein1); cIAP-2 (cellular inhibitor of apoptosis protein2); ILP-2; livin; NAIP (Neuronal Apoptosis Inhibitory Protein); Survivin; and XIAP (X-linked inhibitor of apoptosis protein). Overexpression of these proteins may lead to cell growth and 
tumor survival by suppression of apoptosis. Although not all IAPs are involved in lung cancer, studies have shown possible correlation of IAPS such as, XIAP and survivin in attributing drug resistance in lung cancer. It was demonstrated that XIAP was up-regulated in human H460 NSCLC cell line, thereby leading to decreased activation of the apoptosome, which is an important mediator in caspase-induced cell death. ${ }^{82}$ Similarly, it has been shown that decreased expression of survivin, had significantly better overall survival when compared to patients with higher survivin expression. ${ }^{83}$ However, others have demonstrated no correlation of survivin with patient survival; although they do report that overexpression of survivin is present in early-stage NSCLC ${ }^{84} \mathrm{~A}$ recent study, comparing the expression of livin and survivin mRNA expression by quantitative reverse transcription polymerase chain reaction (RT-PCR) demonstrated a higher expression of both in early-stage NSCLC patients. ${ }^{85}$

Since drugs such as cisplatin, taxol, etoposide and gemcitabine induce tumor cell death by activation of the intrinsic and extrinsic apoptotic pathways, a defect in these pathways would lead to failure to chemotherapeutic treatment. However, there may be an involvement of other factors and proteins involved in the regulation or execution of the apoptotic machinery; such as defects in the mitochondrial electron chain transporters or in the generation of reactive oxygen species, which might contribute to drug resistance. Overall, dysregulation of any of these cell death effector mechanisms may significantly contribute to drug resistance in lung cancer towards many chemotherapy drugs that modify these signal transduction pathways.

\section{Resistance to small molecule inhibitors}

More recently numerous small molecule inhibitors have been developed in an attempt to treat lung cancer patients. Although they have helped in the treatment of patients with primary and metastatic disease, resistance is emerging to these drugs fairly quickly. In an attempt to understand resistance to such inhibitors we have summarized the mechanism of drug resistance against small molecule inhibitors.

Epidermal growth factor receptor (EGFR) is often overexpressed in a wide variety of solid tumors, such as lung, breast, colorectal, and cancers of the head and neck. Binding of ligands such as epidermal growth factor (EGF), transforming growth factor alpha (TGF- $\alpha$ ), heparin-binding EGF (HB-EGF) and betacellulin (BTC) leads to homo- or hetero-dimerization and internalization of the receptor. ${ }^{86}$ Internalization of the receptor leads to phosphorylation of specific tyrosine residues, thereby leading to the activation of downstream signaling cascades. The major signaling pathways that have been shown to be regulated by EGFR activation are the PI3K, MAPK and STAT pathways that lead to increased cell proliferation, angiogenesis, inhibition of apoptosis and cell-cycle progression. Furthermore, higher expression of EGFR has been shown to be associated with increased metastasis, decreased survival and poor prognosis. It has been shown that EGFR overexpression is most frequent in squamous (84\%), large cell (68\%) and adenocarcinoma $(65 \%)$ with very little expression in SCLC ${ }^{87}$ EGFR inhibitors such as monoclonal antibodies (mAb) to EGFR and receptor tyrosine kinase inhibitors (TKIs) have been used in the treatment of NSCLC. Cetuximab $\left(\right.$ Erbitux $\left.^{\circledR}\right)$, a chimeric (mouse/human) mAb binds to the extracellular domain of EGFR, thereby preventing ligand binding to EGFR. This prevents dimerization and autophosphorylation of the receptor, thereby leading to the decreased activation of the downstream signal transduction pathway. In contrast, TKIs such as gefitinib and erlotinib inhibit EGFR phosphorylation and thus the activation of receptor tyrosine kinase.

However, in recent years there have been numerous studies reporting the development of resistance to these EGFR inhibitors. Resistance to EGFR inhibitors can be due to multiple causes such as: mutations in the tyrosine kinase domain; EGFR-independent activation of other signaling transduction pathways; activation of alternative tyrosine kinase receptors; or increased angiogenesis. ${ }^{88,89}$

Studies have shown the presence of EGFR mutation in lung adenocarcinoma in the kinase domain of the EGFR gene. ${ }^{90,91}$ Most of these mutations such as deletions and point mutations (L858R), occur either in exon 19 or exon 21 respectively..$^{92}$ This is of particular interest as tumors with such mutations are highly sensitive to EGFR-TKIs; however they develop resistance to treatment later. It has been shown that the occurrence of point mutations at a second site and/or amplification of the c-Met gene contribute to resistance to these agents. ${ }^{93}$ In a case report study, a patient with advanced, moderately differentiated adenocarcinoma and unresponsive to carboplatin, taxanes and gemcitabine treatment, when treated with gefitinib for 24 months achieved complete remission. However, the disease eventually relapsed in the patient and failed to respond to gefitinib therapy. Transbronchial aspirate and biopsy revealed the presence of a second point mutation in the EGFR gene. The resulting resistance to gefitinib treatment was due to an amino acid change from threonine to methionine at position 790 of EGFR. Thus the occurrence of new mutations in the EGFR 
gene maybe one of the mechanisms involved in patients previously responsive to treatment consequently develop acquired resistance to EGFR inhibitors. ${ }^{94}$

Mutations not only in EGFR but also in KRAS gene can lead to resistance to EGFR inhibitors, such as gefitinib and erlotinib. A study using 60 lung adenocarcinomas that were either refractory or sensitive to the drug showed that KRAS mutations lead to a lack of sensitivity to both gefitinib and erlotinib. Thus, screening patients for KRAS and EGFR mutations prior to the commencement of therapy and others may help to predict the responsiveness of such patients to therapy with EGFR inhibitors..$^{95}$ Examination for EGFR mutations during the course of EGFR inhibitor therapy and on the relapse of the disease will also help in providing alternate therapies.

Apart from mutations in EGFR contributing to resistance to EGFR inhibitors, activation of EGFR-independent pathways can also lead to resistance of EGFR inhibitors. This includes activation of other tyrosine kinase receptors, activation of the PTEN-PI3K-AKT and Raf-Ras-MEK-ERK pathways.

It has been shown that activation of tyrosine kinase receptors, such as insulin-like growth factor receptor-1 (IGF1R), c-met (hepatocyte growth factor receptor) and platelet-derived growth factor (PDGF) leads to increased proliferation and metastasis by activation of similar pathways to that of EGFR. ${ }^{96}$ In a study conducted to understand the correlation of c-met with EGFR resistance, it was shown that the expression of c-met is associated with progressive disease in NSCLC patients. ${ }^{97}$ Moreover, treatment with the c-met antibody DN-30 enhanced gefitinib induced cell inhibition in c-met expressing lung cancer cell lines. Thus, activation of alternative pathways leads to cell survival and tumor growth despite treatment with EGFR inhibitors leading to resistance.

Another important pathway that has been implicated in resistance to EGFR therapy is the PTEN-PI3K-AKT pathway. PTEN, phosphatase and tensin homolog (TEP1), a tumor suppressor protein/3-lipid phosphatase that can lead to decreased tumor growth by regulating Akt activity. Akt promotes cell survival by the inactivation of several targets, such as bad and caspases-9. ${ }^{98}$ Thus, PTEN negatively regulates the PI3KAKT pathway and loss of PTEN leads to decreased tumor growth. ${ }^{99}$ Studies have shown that the loss of PTEN can lead to resistance to EGFR inhibitors such as erlotinib in EGFR mutant lung cancer. ${ }^{100}$ Furthermore, it was shown that H157 lung cancer cells, that express low levels of PTEN, were resistant to gefitinib treatment and the sensitivity to gefitinib was restored after irradiation. ${ }^{101}$ This radiation induced restoration of gefitinib sensitivity is due to a gradual increase of PTEN following radiation treatment. These studies demonstrate the role of PTEN in acquired resistance to EGFR inhibitors.

Increased VEGF expression can also lead to resistance to anti-EGFR drugs, independent of EGFR signaling. Human A341squamous cell carcinoma obtained from severe combined immunodeficiency (SCID) mice xenografts which had undergone treatment with EGFR monoclonal antibodies, $\mathrm{mR} 3$, hR3, or C225; and were resistant to these agents when grown in culture, demonstrated high expression of EGFR. ${ }^{102}$ When analyzed for VEGF expression 5 of the 6 variants exhibited higher VEGF expression. Furthermore, overexpression of VEGF using an expression vector encoding the secreted form of VEGF (VEGF-164), in the A143 variants led to resistance to anti-EGFR antibodies in vivo. Similar results were shown by Ciardiello and colleagues ${ }^{103}$ using human GEO colon cancer cell line in nude mice. Animals treated with EGFR inhibitors after initial regression showed re-growth of the tumor despite continued treatment. However, the treatment of mice with a VEGF flk1/KDR (VEGFR-2) tyrosine kinase inhibitor, ZD6474 led to tumor growth repression.

In conclusion, although EGFR-TKIs are effective in the treatment of lung cancer, resistance to these drugs depends on numerous factors accounted above. One way to overcome or minimize acquired resistance would be to incorporate combination therapies.

Resistance to another small molecule inhibitor reported is against the angiogenesis inhibitor bevacizumab. Angiogenesis is the growth of new blood vessels from preexisting blood vessels. As we know, tumor cells divide and proliferate faster than normal cells and hence they have an increased demand for blood supply, which occurs through angiogenesis. The tumors may secrete various proteins, such as VEGF that promotes the formation of new blood vessels. Since VEGF plays a major role in angiogenesis there has been extensive research and development on drugs that can inhibit angiogenesis by inhibiting VEGF. Often VEGF is secreted by the tumor which then binds to its receptor and leads to the activation of downstream signaling pathways that promote angiogenesis and thus tumor growth and metastasis. To date, three important receptors of VEGF (also known as VEGF-A) have been identified, namely VEGFR-1, VEGFR-2 and VEGFR-3, with VEGFR-2 being the most important in angiogenesis. ${ }^{104}$ Typically the VEGFR has an extracellular portion consisting of 7 immunoglobulin like domains, a single transmembrane region and an intracellular part containing a split tyrosinekinase domain. The VEGF family, is structurally similar 
to platelet derived growth factor and consists of VEGF-A, VEGF-B, VEGF-C, VEGF-D, VEGF-E and placenta growth factor (PlGF). VEGF can not only bind to its counterpart tyrosine kinase receptor known as the VEGF receptor (VEGFR) but can also bind to neuropilin-1(NP1) and neuropilin-2 (NP2), which are transmembrane glycoprotein receptors for another class of proteins known as semaphorins.

Bevacizumab, is a humanized monoclonal antibody that blocks VEGF-A, thereby preventing binding of VEGF-A to its receptor. However, some patients do not respond to bevacizumab therapy. Since bevacizumab blocks VEGF-A, NP1 and NP2 can still augment the effects of VEGFR-1 and VEGFR-2, thereby promoting angiogenesis and activating alternative pathways. Moreover, it has been reported that NP1 and NP2 are expressed in bronchial basal cells in normal lungs and often overexpressed in NSCLC as compared to neuroendocrine tumors. ${ }^{105}$ There have been reports that expression of both NP1 and NP2 was increased in preinvasive lesions that transitioned from hyperplastic mucosa to moderate dysplasia. ${ }^{106}$ Moreover, another study demonstrated that overexpression of neuropilin leads to activation of the MAPK pathway and is associated with resistance to chemotherapy. ${ }^{107}$ Although there are some reports that bevacizumab resistance in breast cancer might be associated with neuropilin, at the present time we have little knowledge in lung cancer. ${ }^{108}$ Thus it is possible that bevacizumab related overexpression of NP1 and NP2 leads to the activation of alternative survival pathways and thus resistance to bevacizumab in lung cancer. However, further investigation would reveal if there are other alternative pathways involved in bevacizumab resistance.

\section{Other mechanisms that play a role in resistance to chemotherapy drugs}

Many more complex pathways and molecules than the ones just discussed also contribute to drug resistance. For example, the tumor-suppressor protein p53 is an important regulator of the cell cycle and activator of various apoptotic pathways. Mutations in TP53, the gene encoding for p53, are found in more than $50 \%$ of cancer types. ${ }^{109}$ Such mutations lead to multiple recurrences of tumors such as those in, breast cancer, brain tumors, acute leukemia, soft tissue and bone sarcomas. Numerous investigators have reported that inactivation or loss of p53 function can lead to resistance to drugs such as 5-fluorouracil and cisplatin. ${ }^{110,111}$ Moreover, most cancer patients with $p 53$ mutations initially respond to chemotherapy but gradually develop resistance to the drugs as the result of a mutation in, or altered function of, certain p53-regulated target genes. ${ }^{112}$
A second example is that patients with NSCLC whose cancer cells express the epidermal growth factor receptor(EGFR) may initially respond well to treatment with anilinoquinazoline EGFR inhibitors such as gefitinib, although they subsequently develop resistance to the drug because of mutations in the EGFR gene. ${ }^{94}$ However, many patients with NSCLC who become resistant to EGFR-targeted drugs have a mutation in only a single building block of the EGFR protein.

Resistance to chemotherapy may also be associated with mutations or alterations in the target of chemotherapeutic drugs such as Paclitaxel. The results from a clinical study conducted in NSCLC patients showed that paclitaxel resistance may be due to a mutation in the $\beta$-tubulin gene. ${ }^{113}$ Paclitaxel binds to the $\beta$-tubulin subunit of the microtubule, thereby stabilizing the microtubules. This paclitaxel/ microtubule complex prevents the microtubules from disassembly during normal cell cycle progression, leading to a cell cycle arrest. However, a mutation in the $\beta$-tubulin gene will lead to structural and/or conformational alteration of the tubulin protein, thereby leading to poor binding of paclitaxel and contributing to paclitaxel resistance. Patients who had $\beta$-tubulin mutations did not respond to paclitaxel therapy and had a median survival of 3 months as compared to median survival of 10 months in patients without such mutations. ${ }^{113}$ In a more recent trial, Yabuki and colleagues demonstrated the role of the $m d r l$ gene in paclitaxel-dependent drug resistance. ${ }^{10}$ Their results demonstrated that the paclitaxel resistant NSCLC cell line H460 created by gradual exposure to paclitaxel had a higher expression of not only the $m d r l$ gene but also of the protein, as compared to the parental cell line. Furthermore, these cell lines were also cross-resistant to other chemotherapy drugs such as docetaxel, vinblastine and doxorubicin.

Additionally, gene mutations in the $\beta$-tubulin gene have been shown to be a predictor of paclitaxel response in NSCLC. A study of 49 patients with NSCLC having mutations in either exon 1 or exon 4 of the $\beta$-tubulin gene, showed no objective response, whereas 13 of 33 patients with no mutations demonstrated complete or partial response to paclitaxel therapy. ${ }^{113}$ Apart from mutations, studies have shown the possible involvement of other proteins, such as, annexin IV, a calcium dependent phospholipid binding protein in resistance to paclitaxel. Treatment of H460 lung cancer cell lines with $10 \mathrm{nM}$ paclitaxel for 4 days led to an increased expression of annexin IV. Overexpression of annexin IV was confirmed by both western blotting and immunostaining thereby demonstrating multiple causes for drug resistance to a particular drug. ${ }^{114}$ 
More recently reports on resistance to proteasome inhibitor therapy are emerging. Proteasome inhibitors such as bortezomib (also known as PS-341) and lactacystin have been used as both chemotherapeutic agents and as agents for overcoming drug resistance. ${ }^{115-120}$ Proteasomes are large multi-subunit cytosolic complexes that are involved in the degradation of ubiquitin-tagged abnormal and misfolded proteins. The $26 \mathrm{~S}$ proteasome is composed of two major subunits; the 20S core subunit and the 19S subunit which flanks the 20S subunit on both sides. The 20S subunit itself is made up of four heptameric $\alpha$ and $\beta$ subunits, but the main proteolytic activity lies within the $\beta 1, \beta 2$, and $\beta 5$ subunits, which cleave acidic, basic, and hydrophobic residues, respectively, owing to their chymotrypsin-like, trypsin-like, and post-glutamyl peptide hydrolyzing-like activities. However, the $\beta 5$ subunit is most important one involved in the degradation of proteins. By inhibiting the $\beta 5$ subunit, proteasome inhibitors are able to induce apoptosis preferentially in tumor cells. $^{121,122}$

Bortezomib was the first proteasome inhibitor that was approved, originally for the treatment of patients with multiple myeloma. Since then it has been shown to act against numerous cancers such as: ovarian; ${ }^{115}$ lung; $;{ }^{116}$ breast; colorectal; and prostate. ${ }^{17,119,123}$ Despite the ability of bortezomib to effectively inhibit tumor growth and restore chemosensitivity to drug-resistant tumors, reports on resistance to bortezomib therapy are coming to light. For example it has been shown that either point mutations or overexpression of the $\beta 5$ proteasomal subunit results in resistance to bortezomib therapy. ${ }^{124,125}$ Preliminary studies in our own laboratory demonstrated cisplatin-resistant lung and ovarian cancer cell lines have reduced proteasome activity and do not respond to proteasome inhibitors effectively when compared to their parental cell lines that are sensitive to cisplatin. Molecular analysis has shown the protein expression of the $\beta 5$ proteasome subunit was greatly reduced in cisplatin-resistant cell lines as compared to their parental counterpart (unpublished data). Associated with reduced $\beta 5$ protein expression was an increased pro- and anti-apoptotic protein expression in cisplatin-resistant cell lines. Overexpression of $\beta 5$ in the cisplatin-resistant cell lines resulted in restoration of proteasome function and sensitization to cisplatin (unpublished data). Our results demonstrate a potential new mechanism for drug resistance where alterations in proteasome function can contribute to resistance to both chemotherapy and proteasome-targeted inhibitors such as bortezomib. We are currently conducting both pre-clinical and clinical investigations to confirm our preliminary findings.

\section{Conclusion}

Our knowledge about drug resistance has increased considerably over the last few decades, but we are still far from having a complete understanding of the underlying mechanisms. Because each tumor type is different at the histologic and molecular levels, it would be difficult to find one mechanism of drug resistance that is common to lung and other cancers. Moreover, each host-specific interaction and tumor microenvironment is unique, so each patient's disease responds differently to the same drugs -some patients may show a response to a particular drug, while others may show complete resistance to the same drug. Furthermore, the coexistence of multiple defects within a cancer cell makes it much more difficult to develop effective therapies. However, with the recent completion of the human genome project and the availability of newly advanced technologies, we anticipate that new mechanisms and targets that contribute to drug resistance will be identified, which in turn will lead to the development and testing of novel therapies.

Additionally, there is a need for the development of new tools to screen patients prior to beginning chemotherapy. More recently a tool known as lung metagene score was developed in an attempt to individualize treatment for lung cancer patients. The lung metagene score (formerly known as the lung metagene predictor) is a screening tool developed to estimate the risk of recurrence in early stage NSCLC. Using microarray analysis, the amount of RNA made from a particular gene is measured. The expression of RNA is recorded and is called the genomic profile of the tissue. It is then compared with other RNA in the database to predict the risk of recurrence. This technique is still under investigation and the data is not available as of yet. Clinical trials and testing, will give the opportunity to identify patients with low recurrence and avoid post-operative chemotherapy, since chemotherapeutic treatment has its own side effects. This will also allow initiation of early and aggressive chemotherapy in patients with a high risk of recurrence in early stage NSCLC. Moreover, such screening tools will also allow us to choose the most appropriate chemotherapy drug that will be most effective in the treatment of such patients, based on their genomic profiling. However, this technique too is still under development and needs extensive clinical trials before it can be used as standard tool in the management of patients with lung cancer.

\section{Acknowledgments}

The authors thank Karen F. Phillips from the Department of Scientific Publications, MD Anderson Cancer Center for editorial assistance. 


\section{Disclosure}

The authors report no conflict of interest relevant to this research.

\section{References}

1. Ambudkar SV, Dey S, Hrycyna CA, Ramachandra M, Pastan I, Gottesman MM. Biochemical, cellular, and pharmacological aspects of the multidrug transporter. Annual Review of Pharmacology and Toxicology. 1999;39:361-398.

2. Townsend DM, Tew KD. The role of glutathione-S-transferase in anti-cancer drug resistance. Oncogene. 2003;22(47):7369-7375.

3. Eastman A, Schulte N. Enhanced Dna-Repair As A Mechanism of Resistance to Cis-Diamminedichloroplatinum(Ii). Biochemistry. 1988;27(13):4730-4734.

4. Kavallaris M, Kuo DYS, Burkhart CA, et al. Taxol-resistant epithelial ovarian tumors are associated with altered expression of specific beta-tubulin isotypes. Journal of Clinical Investigation. 1997;100(5):1282-1293.

5. Sethi T, Rintoul RC, Moore SM, et al. Extracellular matrix proteins protect small cell lung cancer cells against apoptosis: A mechanism for small cell lung cancer growth and drug resistance in vivo. Nature Medicine. 1999;5(6):662-668

6. Green SK, Frankel A, Kerbel RS. Adhesion-dependent multicellular drug resistance. Anticancer Drug Des. 1999;14(2):153-168.

7. Morin PJ. Drug resistance and the microenvironment: nature and nurture. Drug Resistance Updates. 2003;6(4):169-172.

8. Abe Y, Ohnishi Y, Yoshimura M, et al. P-glycoprotein-mediated acquired multidrug resistance of human lung cancer cells in vivo. British Journal of Cancer. 1996;74(12):1929-1934.

9. Volm M, Mattern J, Samsel B. Overexpression of P-Glycoprotein and Glutathione-S-Transferase-Pi in Resistant Non-Small-Cell Lung Carcinomas of Smokers. British Journal of Cancer. 1991;64(4):700-704.

10. Yabuki N, Sakata K, Yamasaki T, et al. Gene amplification and expression in lung cancer cells with acquired paclitaxel resistance. Cancer Genetics and Cytogenetics. 2007;173(1):1-9.

11. Robey RW, Shukla S, Finley EM, et al. Inhibition of P-glycoprotein (ABCB1)- and multidrug resistance-associated protein 1 (ABCC1)mediated transport by the orally administered inhibitor, CBT-1 (R). Biochemical Pharmacology. 2008;75(6):1302-1312.

12. Patil YB, Swaminathan SK, Sadhukha T, Ma L, Panyam J. The use of nanoparticle-mediated targeted gene silencing and drug delivery to overcome tumor drug resistance. Biomaterials. In Press, Corrected Proof.

13. Helfrich B, Chan D, Helm K, Ashton M, Bunn PA. 648 Expression of MDR-1, MRP and LRP in human lung cancer cell lines from previously treated and untreated patients, chemosensitivity of the cell lines and effects of modulators on sensitivity. Lung Cancer. 1997;18 (Supplement 1):166.

14. Berger W, Setinek U, Hollaus $\mathrm{P}$, et al. Multidrug resistance markers P-glycoprotein, multidrug resistance protein 1 , and lung resistance protein in non-small cell lung cancer: prognostic implications. Journal of Cancer Research and Clinical Oncology. 2005;131(6):355-363.

15. Borst P, Evers R, Kool M, Wijnholds J. A family of drug transporters: The multidrug resistance-associated proteins. Journal of the National Cancer Institute. 2000;92(16):1295-1302.

16. Young LC, Campling BG, Cole SPC, Deeley RG, Gerlach JH. Multidrug resistance proteins MRP3, MRP1, and MRP2 in lung cancer: Correlation of protein levels with drug response and messenger RNA levels. Clinical Cancer Research. 2001;7(6):1798-1804.

17. Bessho Y, Oguri T, Ozasa H, et al. ABCC10/MRP7 is associated with vinorelbine resistance in non-small cell lung cancer. Oncology Reports. 2009;21(1):263-268.

18. Awasthi S, Singhal SS, Singhal J, Cheng JH, Zimniak P, Awasthi YC Role of RLIP76 in lung cancer doxorubicin resistance: II. Doxorubicin transport in lung cancer by RLIP76. International Journal of Oncology. 2003;22(4):713-720.
19. Awasthi S, Singhal SS, Singhal J, Yang YS, Zimniak P, Awasthi YC. Role of RLIP76 in lung cancer doxorubicin resistance: III. Anti-RLIP76 antibodies trigger apoptosis in lung cancer cells and synergistically increase doxorubicin cytotoxicity. International Journal of Oncology. 2003;22(4):721-732.

20. Stuckler D, Singhal J, Singhal SS, Yadav S, Awasthi YC, Awasthi S. RLIP76 transports vinorelbine and mediates drug resistance in non-small cell lung cancer. Cancer Research. 2005;65(3):991-998.

21. Scheper RJ, Broxterman HJ, Scheffer GL, et al. Overexpression of A M(R) 110,000 Vesicular Protein in Non-P-Glycoprotein-Mediated Multidrug Resistance. Cancer Research. 1993;53(7):1475-1479.

22. Izquierdo MA, Scheffer GL, Flens MJ, et al. Broad distribution of the multidrug resistance-related vault lung resistance protein in normal human tissues and tumors. American Journal of Pathology. 1996;148(3):877-887.

23. Kitazono M, Sumizawa T, Takebayashi Y, et al. Multidrug resistance and the lung resistance-related protein in human colon carcinoma SW-620 cells. Journal of the National Cancer Institute. 1999;91(19):1647-1653.

24. Dingemans AMC, vanArkOtte J, vanderValk P, et al. Expression of the human major vault protein LRP in human lung cancer samples and normal lung tissues. Annals of Oncology. 1996;7(6):625-630.

25. Kasahara K, Fujiwara Y, Nishio K, et al. Metallothionein Content Correlates with the Sensitivity of Human Small-Cell LungCancer Cell-Lines to Cisplatin. Cancer Research. 1991;51(12): 3237-3242.

26. Kelley SL, Basu A, Teicher BA, Hacker MP, Hamer DH, Lazo JS. Overexpression of Metallothionein Confers Resistance to Anticancer Drugs. Science. 1988;241(4874):1813-1815.

27. Yang M, Chitambar CR. Role of oxidative stress in the induction of metallothionein-2A and heme oxygenase- 1 gene expression by the antineoplastic agent gallium nitrate in human lymphoma cells. Free Radical Biology and Medicine. 2008;45(6):763-772.

28. Klaassen CD, Liu J, Choudhuri S. Metallothionein: An intracellular protein to protect against cadmium toxicity. Annual Review of Pharmacology and Toxicology. 1999;39:267-294.

29. Hoey JG, Garrett SH, Sens MA, Todd JH, Sens DA. Expression of MT-3 mRNA in human kidney, proximal tubule cell cultures, and renal cell carcinoma. Toxicology Letters. 1997;92(2):149-160.

30. Masters BA, Quaife CJ, Erickson JC, et al. Metallothionein-Iii Is Expressed in Neurons That Sequester Zinc in Synaptic Vesicles. Journal of Neuroscience. 1994;14(10):5844-5857.

31. Quaife CJ, Findley SD, Erickson JC, et al. Induction of A New Metallothionein Isoform (Mt-Iv) Occurs During Differentiation of Stratified Squamous Epithelia. Biochemistry. 1994;33(23):7250-7259.

32. Hamer DH. Metallothionein. Annual Review of Biochemistry. 1986;55:913-951.

33. Hishikawa Y, Kohno H, Ueda S, et al. Expression of metallothionein in colorectal cancers and synchronous liver metastases. Oncology. 2001;61(2):162-167.

34. Goulding H, Jasani B, Pereira H, et al. Metallothionein Expression in Human Breast-Cancer. British Journal of Cancer. 1995;72(4): 968-972.

35. Tan Y, Sinniah R, Bay BH, Singh G. Metallothionein expression and nuclear size-in benign, borderline, and malignant serous ovarian tumours. Journal of Pathology. 1999;189(1):60-65.

36. Hishikawa Y, Koji T, Dhar DK, Kinugasa S, Yamaguchi M, Nagasue N. Metallothionein expression correlates with metastatic and proliferative potential in squamous cell carcinoma of the oesophagus. British Journal of Cancer. 1999;81(4):712-720.

37. Janssen AML, van Duijn W, Kubben FJGM, Griffioen G, Lamers CBHW, van Krieken JHJM, et al. Prognostic significance of metallothionein in human gastrointestinal cancer. Clinical Cancer Research. 2002;8(6):1889-1896.

38. Theocharis S, Karkantaris C, Philipides T, et al. Expression of metallothionein in lung carcinoma: correlation with histological type and. Histopathology. 2002;40(2):143-151. 
39. Hishikawa Y,Abe S, Kinugasa S, et al. Overexpression of metallothionein correlates with chemoresistance to cisplatin and prognosis in esophageal cancer. Oncology. 1997;54(4):342-347.

40. Vandier D, Calvez V, Massade L, et al. Transactivation of the metallothionein promoter in cisplatin-resistant cancer cells: a specific gene therapy strategy. Journal of the National Cancer Institute. 2000;92(8):642-647.

41. Mattern J, Volm M. Increased Resistance to Doxorubicin in Human Non-Small-Cell Lung Carcinomas with Metallothionein Expression. International Journal of Oncology. 1992;1(6):687-689.

42. Tew KD. Glutathione-Associated Enzymes in Anticancer DrugResistance. Cancer Research. 1994;54(16):4313-4320.

43. Awasthi YC, Singh SV, Ahmad H, Moller PC. Immunocytochemical Evidence for the Expression of Gst1, Gst2, and Gst3 Gene Loci for GlutathioneS-Transferase in Human-Lung. Lung. 1987;165(6):323-332.

44. Diilio C, Delboccio G, Aceto A, Casaccia R, Mucilli F, Federici G. Elevation of Glutathione Transferase-Activity in Human-Lung Tumor. Carcinogenesis. 1988;9(2):335-340.

45. Buller AL, Clapper ML, Tew KD. Glutathione S-Transferases in Nitrogen Mustard-Resistant and Mustard-Sensitive Cell-Lines. Molecular Pharmacology. 1987;31(6):575-578.

46. Nakagawa K, Saijo N, Tsuchida S, et al. Glutathione-S-Transferase-Pi As A Determinant of Drug-Resistance in Transfectant Cell-Lines. Journal of Biological Chemistry. 1990;265(8):4296-4301.

47. Linsenmeyer ME, Jefferson S, Wolf M, Matthews JP, Board PG, Woodcock DM. Levels of Expression of the Mdr1 Gene and GlutathioneS-Transferase Gene-2 and Gene-3 and Response to Chemotherapy in Multiple-Myeloma. British Journal of Cancer. 1992;65(3):471-475.

48. Ilizarov AM, Koo HC, Kazzaz JA, et al. Overexpression of manganese superoxide dismutase protects lung epithelial cells against oxidant injury. American Journal of Respiratory Cell and Molecular Biology. 2001;24(4):436-441.

49. Kinnula K, Linnainmaa K, Raivio KO, Kinnula VL. Endogenous antioxidant enzymes and glutathione S-transferase in protection of mesothelioma cells against hydrogen peroxide and epirubicin toxicity. British Journal of Cancer. 1998;77(7):1097-1102.

50. Kinnula VL, PietarinenRuntti P, Raivio K, et al. Manganese superoxide dismutase in human pleural mesothelioma cell lines. Free Radical Biology and Medicine. 1996;21(4):527-532.

51. Barabas K, Milner R, Lurie D, Adin C. Cisplatin: a review of toxicities and therapeutic applications. Veterinary and Comparative Oncology. 2008;6(1):1-18.

52. Gossage L, Madhusudan S. Current status of excision repair cross complementing-group 1 (ERCC1) in cancer. Cancer Treatment Reviews. 2007;33(6):565-577.

53. Steffensen KD, Waldstrom M, Jakobsen A. The Relationship of Platinum Resistance and ERCC1 Protein Expression in Epithelial Ovarian Cancer. International Journal of Gynecological Cancer. 2009;19(5):820-825.

54. Schettino C, Bareschino MA, Maione P, Rossi A, Ciardiello F, Gridelli C. The potential role of pharmacogenomic and genomic in the adjuvant treatment of early stage non small cell lung cancer. Current Genomics. 2008;9(4):252-262.

55. Rosell R, Lord RVN, Taron M, Reguart N. DNA repair and cisplatin resistance in non-small-cell lung cancer. Lung Cancer. 2002;38(3): 217-227.

56. Lord RVN, Brabender J, Gandara D, et al. Low ERCC1 expression correlates with prolonged survival after cisplatin plus gemcitabine chemotherapy in non-small cell lung cancer. Clinical Cancer Research. 2002;8(7):2286-2291.

57. Li GM. Mechanisms and functions of DNA mismatch repair. Cell Research. 2008;18(1):85-98.

58. Liu A, Yoshioka KI, Salerno V, Hsieh P. The mismatch repair-mediated cell cycle checkpoint response to fluorodeoxyuridine. Journal of Cellular Biochemistry. 2008;105(1):245-254.

59. Preston TJ, Henderson JT, McCallum GP, Wells PG. Base excision repair of reactive oxygen species-initiated 7,8-dihydro-8-oxo-2 '-deoxyguanosine inhibits the cytotoxicity of platinum anticancer drugs. Molecular Cancer Therapeutics. 2009;8(7):2015-2026.
60. Topping RP, Wilkinson JC, Scarpinato KD. Mismatch Repair Protein Deficiency Compromises Cisplatin-induced Apoptotic Signaling. Journal of Biological Chemistry. 2009;284(21):14029-14039.

61. Adhikari S, Choudhury S, Mitra PS, Dubash JJ, Sajankila SP, Roy R. Targeting base excision repair for chemosensitization. Anti-Cancer Agents in Medicinal Chemistry. 2008;8(4):351-357.

62. Robertson AB, Klungland A, Rognes T, Leiros I. DNA Repair in Mammalian Cells. Cellular and Molecular Life Sciences. 2009;66(6):981-993.

63. Viktorsson K, De Petris L, Lewensohn R. The role of p53 in treatment responses of lung cancer. Biochemical and Biophysical Research Communications. 2005;331(3):868-880.

64. Hefferin ML, Tomkinson AE. Mechanism of DNA double-strand break repair by non-homologous end joining. Dna Repair. 2005;4(6): 639-648.

65. Pastwa E, Poplawski T, Czechowska A, Malinowski M, Blasiak J. Non-homologous DNA end joining repair in normal and leukemic cells depends on the substrate ends. Zeitschrift fur Naturforschung $C$ - $A$ Journal of Biosciences. 2005;60(5-6):493-500.

66. Polischouk AG, Holgersson A, Zong DL, et al. The antipsychotic drug trifluoperazine inhibits DNA repair and sensitizes non-small cell lung carcinoma cells to DNA double-strand break-induced cell death. Molecular Cancer Therapeutics. 2007;6(8):23032309.

67. Friesen C, Uhl M, Pannicke U, Schwarz K, Miltner E, Debatin KM. DNA-ligase IV and DNA-protein kinase play a critical role in deficient caspases activation in apoptosis-resistant cancer cells by using doxorubicin. Molecular Biology of the Cell. 2008;19(8):3283-3289.

68. Shrivastav M, De Haro LP, Nickoloff JA. Regulation of DNA doublestrand break repair pathway choice. Cell Research. 2008;18(1): 134-147.

69. Miyagawa K. Clinical relevance of the homologous recombination machinery in cancer therapy. Cancer Science. 2008;99(2):187-194.

70. Niedernhofer LJ, Essers J, Weeda G, et al. The structure-specific endonuclease Ercc1-Xpf is required for targeted gene replacement in embryonic stem cells. Embo Journal. 2001;20(22):6540-6549.

71. Hansen LT, Lundin C, Spang-Thomsen M, Petersen LN, Helleday T. The role of RAD51 in etoposide (VP16) resistance in small cell lung cancer. International Journal of Cancer. 2003;105(4):472-479.

72. Hansen LT, Lundin C, Helleday T, et al. DNA repair rate and etoposide (VP16) resistance of tumor cell subpopulations derived from a single human small cell lung cancer. Lung Cancer. 2003;40(2): $157-164$.

73. Sigmond J, Backus HHJ, Wouters D, Temmink OH, Jansen G, Peters GJ. Induction of resistance to the multitargeted antifolate Pemetrexed (ALIMTA) in WiDr human colon cancer cells is associated with thymidylate synthase over expression. Biochemical Pharmacology. 2003;66(3):431-438.

74. Peters GJ, Backus HHJ, Freemantle S, et al. Induction of thymidylate synthase as a 5-fluorouracil resistance mechanism. Biochimica et Biophysica Acta-Molecular Basis of Disease. 2002;1587(2-3): 194-205.

75. Lin L, Liu X, Rao Y, Wang W, Wang S, Song S. Detection of gemcitabine-resistant genes expression in lung cancer cell lines using real-time PCR. The Chinese-German Journal of Clinical Oncology. 2008;7(12):682-685.

76. Biswas RS, Cha HJ, Hardwick JM, Srivastava RK. Inhibition of drug-induced Fas ligand transcription and apoptosis by Bcl-X-L. Molecular and Cellular Biochemistry. 2001;225(1-2):7-20.

77. Srivastava RK, Sasaki CY, Hardwick JM, Longo DL. Bcl-2-mediated drug resistance: Inhibition of apoptosis by blocking nuclear factor of activated T lymphocytes (NFAT)-induced Fas ligand transcription. Journal of Experimental Medicine. 1999;190(2):253265 .

78. Inoue $\mathrm{Y}$, Gika $\mathrm{M}, \mathrm{Abiko} \mathrm{T}$, et al. Bcl-2 overexpression enhances in vitro sensitivity against docetaxel in non-small cell lung cancer. Oncology Reports. 2005;13(2):259-264. 
79. Los M, Herr I, Friesen C, Fulda S, SchulzeOsthoff K, Debatin KM. Cross-resistance of CD95- and drug-induced apoptosis as a consequence of deficient activation of caspases (ICE/Ced-3 proteases). Blood. 1997;90(8):3118-3129.

80. Okouoyo S, Herzer K, Ucur E, et al. Rescue of death receptor and mitochondrial apoptosis signaling in resistant human NSCLC in vivo. International Journal of Cancer. 2004;108(4):580-587.

81. Volm M, Mattern J, Koomagi R. Inverse correlation between apoptotic (Fas ligand, caspase-3) and angiogenic factors (VEGF, microvesse density) in squamous cell lung carcinomas. Anticancer Research. 1999;19(3A):1669-1671.

82. Yang LL, Mashima T, Sato S, et al. Predominant suppression of apoptosome by inhibitor of apoptosis protein in non-small cell lung cancer H460 cells: Therapeutic effect of a novel polyarginine-conjugated Smac peptide. Cancer Research. 2003;63(4):831-837.

83. Monzo M, Rosell R, Felip E, et al. A novel anti-apoptosis gene: Re-expression of survivin messenger RNA as a prognosis marker in non-small-cell lung cancers. Journal of Clinical Oncology. 1999;17(7):2100-2104.

84. Falleni M, Pellegrini C, Marchetti A, et al. Survivin gene expression in early-stage non-small cell lung cancer. Journal of Pathology. 2003;200(5):620-626.

85. Tanabe H, Yagihashi A, Tsuji N, Shijubo Y, Abe S, Watanabe N. Expression of survivin mRNA and livin mRNA in non-small-cell lung cancer Lung Cancer. 2004;46(3):299-304.

86. Salomon DS, Brandt R, Ciardiello F, Normanno N. Epidermal Growth Factor-Related Peptides and Their Receptors in Human Malignancies. Critical Reviews in Oncology/Hematology. 1995;19(3): 183-232.

87. Bunn PA, Franklin W. Epidermal growth factor receptor expression, signal pathway, and inhibitors in non-small cell lung cancer. Seminars in Oncology. 2002;29(5):38-44.

88. Bianco R, Troiani T, Tortora G, Ciardiello F. Intrinsic and acquired resistance to EGFR inhibitors in human cancer therapy. EndocrineRelated Cancer. 2005;12:S159-S171.

89. Camp ER, Summy J, Bauer TW, Liu WB, Gallick GE, Ellis LM. Molecular mechanisms of resistance to therapies targeting the epidermal growth factor receptor. Clinical Cancer Research. 2005;11(1):397-405.

90. Paez JG, Janne PA, Lee JC, et al. EGFR mutations in lung cancer: Correlation with clinical response to gefitinib therapy. Science. 2004;304(5676):1497-1500.

91. Lynch TJ, Bell DW, Sordella R, et al. Activating mutations in the epidermal growth factor receptor underlying responsiveness of non-small-cell lung cancer to gefitinib. New England Journal of Medicine. 2004;350(21):2129-2139.

92. Mitsudomi T, Yatabe Y. Mutations of the epidermal growth factor receptor gene and related genes as determinants of epidermal growth factor receptor tyrosine kinase inhibitors sensitivity in lung cancer. Cancer Science. 2007;98(12):1817-1824

93. Engelman JA, Zejnullahu K, Mitsudomi T, et al. MET amplification leads to gefitinib resistance in lung cancer by activating ERBB3 signaling. Science. 2007;316(5827):1039-1043.

94. Kobayashi S, Boggon TJ, Dayaram T, et al. EGFR mutation and resistance of non-small-cell lung cancer to gefitinib. New England Journal of Medicine. 2005;352(8):786-792

95. Pao W, Wang TY, Riely GJ, et al. KRAS mutations and primary resistance of lung adenocarcinomas to gefitinib or erlotinib. Plos Medicine. 2005;2(1):57-61.

96. Leroith D, Werner H, Beitnerjohnson D, Roberts CT. Molecular and Cellular Aspects of the Insulin-Like Growth-Factor-I Receptor. Endocrine Reviews. 1995;16(2):143-163.

97. Zucali PA, Ruiz MG, Giovannetti E, et al. Role of cMET expression in non-small-cell lung cancer patients treated with EGFR tyrosine kinase inhibitors. Annals of Oncology. 2008;19(9):1605-1612.

98. Cardone MH, Roy N, Stennicke HR, et al. Regulation of cell death protease caspase-9 by phosphorylation. Science. 1998;282(5392): $1318-1321$
99. Cantley LC, Neel BG. New insights into tumor suppression: PTEN suppresses tumor formation by restraining the phosphoinositide 3 kinase AKT pathway. Proceedings of the National Academy of Sciences of the United States of America. 1999;96(8):4240-4245.

100. Sos ML, Koker M, Weir BA, et al. PTEN Loss Contributes to Erlotinib Resistance in EGFR-Mutant Lung Cancer by Activation of Akt and EGFR. Cancer Research. 2009;69(8):3256-3261.

101. Zhuang HQ, Wang J, Yuan ZY, Zhao LJ, Wang P, Wang CL. The drugresistance to gefitinib in PTEN low expression cancer cells is reversed by irradiation in vitro. Journal of Experimental and Clinical Cancer Research. 2009;28.

102. Viloria-Petit A, Crombet T, Jothy S, et al. Acquired resistance to the antitumor effect of epidermal growth factor receptor-blocking antibodies in vivo: A role for altered tumor angiogenesis. Cancer Research. 2001;61(13):5090-5101.

103. Ciardiello F, Bianco R, Caputo R, et al. Antitumor activity of ZD6474, a vascular endothelial growth factor receptor tyrosine kinase inhibitor, in human cancer cells with acquired resistance to antiepidermal growth factor receptor therapy. Clinical Cancer Research. 2004;10(2):784-793.

104. Holmes K, Roberts OL, Thomas AM, Cross MJ. Vascular endothelial growth factor receptor-2: Structure, function, intracellular signalling and therapeutic inhibition. Cellular Signalling. 2007;19(10):2003 2012.

105. Lantuejoul S, Constantin B, Drabkin H, Brambilla C, Roche J, Brambilla E. Expression of VEGF, semaphorin SEMA3F, and their common receptors neuropilins NP1 and NP2 in preinvasive bronchial lesions, lung tumours, and cell lines. Journal of Pathology. 2003;200(3):336-347.

106. Roche J, Drabkin H, Brambilla E. Neuropilin and its ligands in normal lung and cancer. Neuropilin: from Nervous System to Vascular and Tumor Biology. 2002;515:103-114.

107. Wey JS, Gray MJ, Fan F, et al. Overexpression of neuropilin-1 promotes constitutive MAPK signalling and chemoresistance in pancreatic cancer cells. British Journal of Cancer. 2005;93(2):233-241.

108. Staton CA, Yang Z, Reed MWR, Brown NJ. Bevacizumab resistance in breast cancer: are neuropilins the key? Breast Cancer Research. 2008;10:S38

109. Levine AJ. p53, the cellular gatekeeper for growth and division. Cell. 1997;88(3):323-331.

110. Fan SJ, Eldeiry WS, Bae I, et al. P53 Gene-Mutations Are Associated with Decreased Sensitivity of Human Lymphoma-Cells to DnaDamaging Agents. Cancer Research. 1994;54(22):5824-5830.

111. Bunz F, Hwang PM, Torrance C, et al. Disruption of p53 in human cancer cells alters the responses to therapeutic agents. Journal of Clinical Investigation. 1999;104(3):263-269.

112. Shelling AN. Role of p53 in drug resistance in ovarian cancer. Lancet. 1997;349(9054):744-745.

113. Monzo M, Rosell R, Sanchez JJ, et al. Paclitaxel resistance in non-small-cell lung cancer associated with beta-tubulin gene mutations. Journal of Clinical Oncology. 1999;17(6):1786-1793.

114. Han EKH, Tahir SK, Cherian SP, Collins N, Ng SC. Modulation of paolitaxel resistance by annexin IV in human cancer cell lines. British Journal of Cancer. 2000;83(1):83-88.

115. Frankel A, Man S, Elliott P, Adams J, Kerbel RS. Lack of multicellular drug resistance observed in human ovarian and prostate carcinoma treated with the proteasome inhibitor PS-341. Clinical Cancer Research. 2000;6(9):3719-3728.

116. Ling YH, Liebes L, Jiang JD, et al. Mechanisms of proteasome inhibitor PS-341-induced G(2)-M-phase arrest and apoptosis in human non-small cell lung cancer cell lines. Clinical Cancer Research. 2003;9(3):1145-1154.

117. Teicher BA, Ara G, Herbst R, Palombella VJ, Adams J. The proteasome inhibitor PS-341 in cancer therapy. Clinical Cancer Research. 1999;5(9):2638-2645.

118. Cusack JC, Liu R, Houston M, et al. Enhanced chemosensitivity to CPT11 with proteasome inhibitor PS-341: Implications for systemic nuclear factor-kappa B inhibition. Cancer Research. 2001;61(9):3535-3540. 
119. Adams J, Palombella VJ, Sausville EA, et al. Proteasome inhibitors: A novel class of potent and effective antitumor agents. Cancer Research. 1999;59(11):2615-2622.

120. Ogiso Y, Tomida A, Lei SH, Omura S, Tsuruo T. Proteasome inhibition circumvents solid tumor resistance to topoisomerase II-directed drugs. Cancer Research. 2000;60(9):2429-2434.

121. Bonvini P, Zorzi E, Basso G, Rosolen A. Bortezomib-mediated 26S proteasome inhibition causes cell-cycle arrest and induces apoptosis in CD-30(+) anaplastic large cell lymphoma. Leukemia. 2007;21(4): $838-842$.

122. Chondrogianni N, Gonos ES. Overexpression of proteasome beta 5 subunit increases amount of assembled proteasome and confers ameliorated response to oxidative stress and higher survival rates. Febs Journal. 2005;272:151.

123. Ling YH, Liebes L, Jiang JD, et al. Mechanisms of proteasome inhibitor PS-341-induced G(2)-M-phase arrest and apoptosis in human non-small cell lung cancer cell lines. Clinical Cancer Research. 2003;9(3):1145-1154.
124. Lu SQ, Yang JM, Song XM, et al. Point mutation of the proteasome beta 5 subunit gene is an important mechanism of bortezomib resistance in bortezomib-selected variants of Jurkat $\mathrm{T}$ cell lymphoblastic lymphoma/leukemia line. Journal of Pharmacology and Experimental Therapeutics. 2008;326(2):423-431.

125. Oerlemans R, Franke NE, Assaraf YG, et al. Molecular basis of bortezomib resistance: proteasome subunit beta 5 (PSMB5) gene mutation and overexpression of PSMB5 protein. Blood. 2008;112(6):2489-2499.

\section{Publish your work in this journal}

Lung Cancer: Targets and Therapy is an international, peer-reviewed, open access journal focusing on lung cancer research, identification of therapeutic targets and the optimal use of preventative and integrated treatment interventions to achieve improved outcomes, enhanced survival and quality of life for the cancer patient. Specific topics covered in the journal include:
Epidemiology, detection and screening; Cellular research and biomarkers; Identification of biotargets and agents with novel mechanisms of action; Optimal clinical use of existing anticancer agents, including combination therapies; Radiation and surgery; Palliative care; Patient adherence, quality of life, satisfaction; Health economic evaluations.

Submit your manuscript here: http://www.dovepress.com/lung-cancer-targets--therapy-journal 